Génét. Sél. Evol., 1985, 17 (4), 579-584

\title{
Note
}

\section{Séquences homologues à l'élément P chez des espèces de Drosophila du groupe obscura et chez Scaptomyza pallida (Drosophilidae).}

\author{
D. ANXOLABEHERE, Danielle NOUAUD et G. PERIQUET * \\ Laboratoire de Génétique des Populations, Mécanismes moléculaires de la Spéciation, \\ Tour 42, Université Paris VI, 2, place Jussieu, F 75005 Paris \\ * Institut de Biocénotique expérimentale des Agrosystèmes \\ Université de Tours, Parc Grandmont, F 37200 Tours
}

\section{Résumé}

Des séquences homologues à l'élément $\mathrm{P}$ de $D$. melanogaster ont été trouvées dans plusieurs espèces du groupe obscura ainsi que dans le genre proche Scaptomyza ( $s p$. pallida). Ces résultats suggèrent une très grande ancienneté de ce type d'éléments parmi les membres de la radiation Sophophora.

Mots clés : Eléments transposables, évolution, phylogénie, Drosophila.

\section{Summary \\ $P$ homologous sequences in Drosophila species of obscura group and in Scaptomyza pallida (Drosophilidae)}

Sequences with homology to the $D$. melanogaster $P$ element were found in many species of the obscura group and in the closely related genus Scaptomyza (pallida sp.). These results suggest that $P$ sequences have a long evolutionary history in the Sophophoran radiation.

Key words : Transposuble elements, evolution, phylogeny, Drosophila.

\section{Introduction}

Chez les Drosophilidés, la distribution phylogénétique de plusieurs familles d'éléments d'ADN moyennement répété révèle que l'évolution de ces séquences diffère de celle des séquences uniques. Au cours de la diversification de ce taxon, certaines familles d'éléments répétés seraient apparues ou auraient disparu (MARTIN et al., 1983). Chez $D$. melanogaster, la famille des éléments $\mathrm{P}$ est constituée d'un groupe hétérogène 
de séquences d'ADN mobilisables, pouvant générer un ensemble d’anomalies décrites dans le syndrome de la dysgénésie des hybrides (KIDWELl et al., 1977 ; revue in ENGELS, 1983). Les éléments $P$ autonomes correspondent à des séquences d'ADN de 2,9 Kb (OHARE \& Rubin, 1983). La famille des éléments $\mathrm{P}$ est également formée de séquences plus courtes, issues de l'élément complet par délétions internes.

L'absence de séquences homologues à $P$ dans les autres espèces du sous-groupe melanogaster (BROOKFIEld et al., 1984) a suggéré l'hypothèse d'une contamination par un transfert horizontal (ENGELS, 1983). La présence de séquences homologues chez les 6 semi-espèces de $D$. paulistorum et dans de nombreuses autres espèces des groupes willistoni et saltans (Daniels et al., 1984 ; Daniels \& Strausbaugh, 1985) a renforcé cette hypothèse et a incité à voir dans ces groupes l'origine possible d'un tel transfert. La mise en évidence de séquences $P$ homologues dans d'autres groupes de drosophilidés repose le problème de l'origine de la présence de ces éléments chez D. melanogaster.

\section{Matériel et méthodes}

Neuf espèces du groupe obscura ont été analysées. Les échantillons étudiés ont été capturés récemment (moins de 5 ans) dans des populations européennes, et ont été depuis élevées en laboratoire. L'espèce Scaptomyza palida a été capturée en 1984 et 1985 en France et en Espagne et analysée, soit directement, soit après quelques générations d'élevage en laboratoire.

La recherche de séquences homologues à l'élément $\mathrm{P}$ a été réalisée par la technique du squash blot (TCHEN et al., 1985) permettant d'analyser séparément les individus. Chaque espèce a été étudiée sur 25 individus, écrasés sur un filtre de nylon (Biodyne). Après traitement selon cette technique, les filtres sont hybridés avec une sonde marquée au ${ }^{32} \mathrm{P}$, puis autoradiographiés. Les 2 sondes utilisées (voir figure) sont des fragments de restriction internes à l'élément $P$ présent sur le plasmide $\mathrm{p} \pi 25.1$ (sonde $S 1$ : fragment Hind III, sonde S2 : fragment Hind III-Sal I). Elles représentent ensemble 80 p. 100 de l'élément $P$. Les conditions d'hybridation et de rinçage sont identiques à celles décrites par AnXolabehere et al. (1985). Les individus témoins proviennent de différentes souches de $D$. melanogaster: Harwich (présence de 30 à 50 copies de P), singed weak (présence d'une à deux copies), Gruta (absence d'éléments P).

\section{Résultats et discussion}

Les résultats sont illustrés par la figure et résumés dans le tableau. Huit des 9 espèces du groupe obscura analysées révèlent la présence de séquences homologues à $P$. Les signaux d'hybridation présentent, pour une sonde donnée, des intensités similaires lorsque l'on compare des individus d'une même espèce, mais montrent de très grandes différences lorsque l'on compare les espèces entre elles. Cependant, dans tous les cas, l'intensité du signal est plus faible que celle obtenue avec la souche Harwich de $D$. melanogaster. Cette diminution d'intensité peut être attribuée soit à une différence d'homologie ou de structure entre les séquences, conduisant à une hybridation plus labile, soit à une différence dans le nombre moyen de copies présentes dans chacune 


\section{Elément $P$}
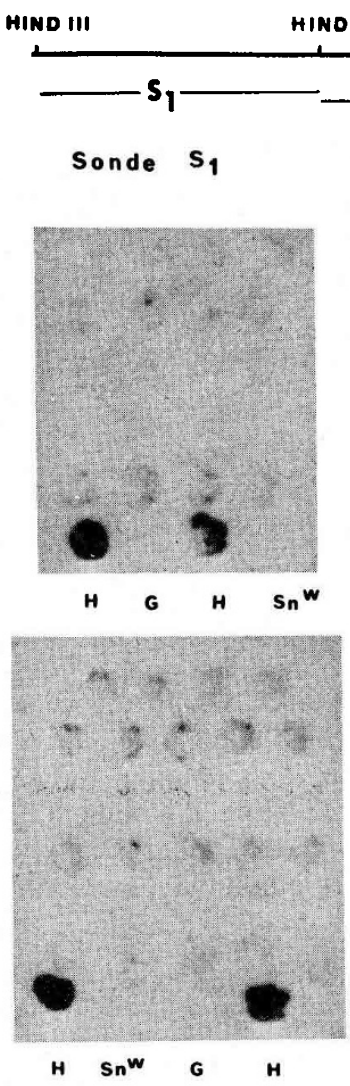

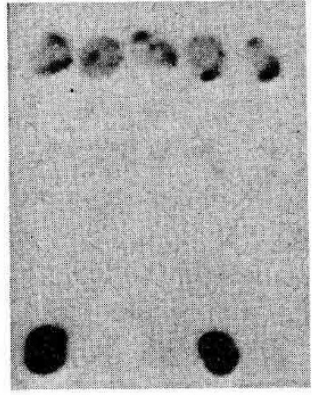

H G Snw $H$
D. subobscura'
D. sub obscura ${ }^{2}$

D. pseudoobscura

D. obscura

D. bifasciata'

TEMOINS

D. helvetica

D. bifasciata ${ }^{2}$

D. a mbigua

D. bifasciata ${ }^{3}$

D.tristis

D. madeirensis

TEMOINS

$s_{2}$

SAL I
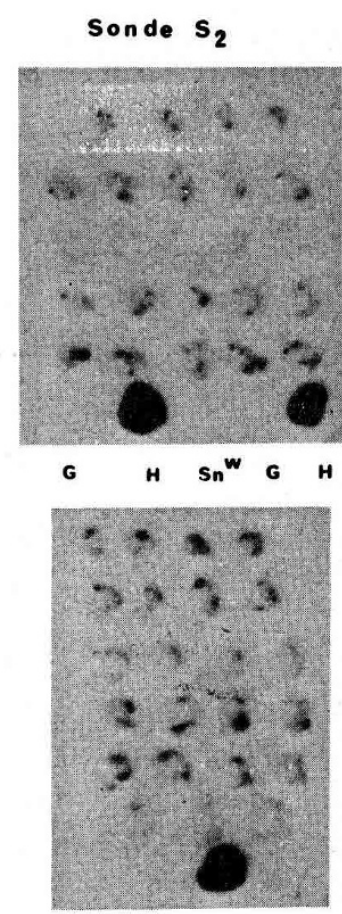

G H $S n$ w

D. guanche

D. $t$ s ac a si

D. tsac as i

D. eugrasilis

D. I gans

D. ficusphila

TEMOINS

\section{FIGURE}

Recherche d'homologies de séquence avec l'élément $P$ par la méthode du squash blot dans des espèces de Drosophiles des groupes obscura et melanogaster. $P$ element homologous sequences in different species of Drosophila among obscura and melanogaster groups.

(1-2-3 : sont des populations d'origine géographique différentes).

(1-2-3: correspond to populations from different geographical area). 


\section{TABLEAU}

Présence de séquences homologues aux sondes $S 1$ et $S 2$ de l'élément $P$ de $\mathrm{D}$. melanogaster chez diverses espèces du groupe obscura.

Homologous sequences of the $S 1$ and $S 2$ probes from the $P$ element of D. melanogaster among species of the obscura group.

\begin{tabular}{|c|c|c|}
\hline Espèces & Sonde S1 & Sonde S2 \\
\hline D. guanche & + & - \\
\hline D. madeirensis . . & + & + \\
\hline D. subobscura $\ldots \ldots \ldots \ldots \ldots \ldots \ldots$ & + & + \\
\hline D. obscura $\ldots \ldots \ldots \ldots \ldots \ldots \ldots$ & - & + \\
\hline D. ambigua $\ldots \ldots \ldots \ldots \ldots \ldots$ & - & + \\
\hline D. tristis $_{\ldots \ldots \ldots \ldots \ldots \ldots \ldots \ldots \ldots \ldots}$ & - & + \\
\hline D. bifasciata $\ldots \ldots \ldots \ldots \ldots \ldots$ & + & + \\
\hline D. pseudoobscura $\ldots \ldots \ldots \ldots \ldots \ldots$ & $+(?)$ & $+(?)$ \\
\hline D. helvetica $\ldots \ldots \ldots \ldots \ldots \ldots \ldots$ & + & + \\
\hline
\end{tabular}

des espèces. Ces 2 interprétations sont en fait non exclusives. La différence de structure est bien illustrée par les résultats obtenus chez $D$. guanche d'une part et chez $D$. obscura, $D$. ambigua et $D$. tristis d'autre part. Dans le premier cas, l'homologie de séquences n'est révélée qu'avec la sonde $\mathrm{S} 1$ tandis qu'à l'inverse, seule la sonde S2 permet de révéler des séquences homologues chez les 3 autres espèces. Chez $D$. pseudoobscura, l'intensité du signal est à la limite du pouvoir de résolution de la technique et ne permet pas de conclure.

La présence de séquences homologues à l'élément $\mathbf{P}$ apparaît ainsi largement répandu dans le groupe obscura, et plus précisément dans le sous-groupe obscura. $D$. helvetica étant la seule espèce du sous-groupe affinis analysée dans le présent travail, on ne peut généraliser ces résultats à l'ensemble de ce sous-groupe. Par ailleurs, 24 autres espèces de drosophilidés ont été analysées, 22 d'entre elles ont donné des résultats négatifs (liste ci-dessous).

Drosophila, sg. Sophophora, gr. melanogaster :

- ss gr melanogaster : D. simulans,D. orena, D. erecta, D. teissieri, D. yakuba, D. mauritiana

- ss gr montium : D. bocqueti, D. burlai, D. kikkawai, D. vulkana

- ss gr ananassae : D. ananassae, D. malerkotliana

- ss gr eugrasilis : D. eugrasilis

- ss gr elegans : D. elegans

— ss gr ficusphila: D. ficusphila

sg. Drosophila : gr. immigrans : D. immigrans

gr cardini : D. cardini, D. arawakana

gr. repleta : D. hydei, D. repleta

sg. Dorsilopha : D. busckii

Zaprionus, Zaprionus collarti 
Par contre, D. tsacasi, du sous-groupe montium, hybride faiblement. Ce résultat est intéressant car il montre que $D$. melanogaster n'est pas la seule espèce du groupe melanogaster à posséder des éléments $\mathrm{P}$ et que certaines séquences homologues sont présentes dans au moins une autre espèce de ce groupe. L'autre cas d'hybridation positive est celui observé chez Scaptomyza pallida et ceci dans 3 populations de cette espèce : Bordils (Espagne), Alès et Tours (France). Le fait qu'il s'agisse d'un autre genre de la famille des drosophilidés montre la large répartition de ces séquences au sein de ce taxon. En l'état actuel de nos connaissances, l'élément $\mathbf{P}$ est connu uniquement dans des groupes taxonomiques appartenant aux sous genre Sophophora (melanogaster, obscura, willistoni et saltans) à l'exception de Scaptomyza pallida. Cependant, les données phylogénétiques (ThrocKMorToN, 1975; HACKMAN, 1982) suggèrent que le genre Scaptomyza pourrait être proche de la radiation qui a produit le sous genre Sophophora du genre Drosophila. Il apparaît ainsi que ces séquences sont largement répandues parmi certaines espèces de la radiation Sophophora.

Deux interprétations sont possibles : l'hypothèse de transmissions horizontales multiples mais restreintes aux espèoes de ce sous genre paraît peu vraisemblable compte tenu de la possibilité de transférer expérimentalement l'élément $\mathbf{P}$ dans des espèces très différentes (BRENNAN et al., 1984). Les résultats présents sont plutôt en faveur d'une grande ancienneté (plus de 10 millions d'années) de ce type de séquences au sein de la radiation Sophophora, et peut-être même de leur présence dès l'origine de cette radiation. Ces séquences auraient été généralement maintenues dans les diverses lignées évolutives de cette radiation, mais quelquefois perdues lors de processus de spéciation dont certaines pourraient être liées aux propriétés dysgénésiques de l'élément $P$. Les espèces ainsi dépourvues de ces séquences auraient pu les acquérir ultérieurement par un phénomène de transfert horizontal. Cela semble être actuellement le cas chez D. melanogaster (ANXOLABEHERE et al., 1982, 1984, 1985 ; KidWELL, 1983). Ces travaux suggèrent que le phénomène a pu se produire à partir d'une zone méridionale de l'Amérique du nord ou centro-américaine. Les résultats du présent travail montrent en fait que les éléments $\mathbf{P}$ actuels de $D$. melanogaster peuvent provenir de l'une des nombreuses espèces américaines de Drosophila ou de Scaptomyza possédant des séquences homologues.

\section{Remerciements}

Nous remercions J. David pour ses commentaires sur le manuscrit, Y. Carton, C. Krimbas, F. Lemeunier, M. Monclus, A. Prevosti, G. Ribo et L. Tsackas pour l'obtention et l'identification des espèces analysées.

Reçu le 28 août 1985.

Accepté le 18 septembre 1985.

\section{Références bibliographiques}

Anxolabehere D., Nouaud D., Périquet G., 1892. Etude de la variabilité du système P-M de dysgénésie des hybrides entre populations de Drosophila melanogaster. C.R. Acad. Sci., Paris, 294, 913-918. 
Anxolabehere D., Kai H., Nouaud D., Périquet G., Ronsseray S., 1984. The geographical distribution of P-M hybrid dysgenesis in Drosophila melanogaster. Genet. Sel. Evol., 16, 15-26.

Anxolabehere D., Nouaud D., Periquet G., Tchen P., 1985. P-element distribution in Eurasian populations of Drosophila melanogaster : a genetic and molecular analysis. Proc. Nat. Acad. Sc., (U.S.A.), 82, 5418-5422.

Brennan M.D., Rowan R.G., Dickinson W.J., 1984. Introduction of a fonctional P element into the germ-line of Drosophila hawaiiensis. Cell, 38, 147-151.

Brookfield J.F.Y., Montgomery E., LANGLeY C.H., 1984. Lack of P element homology in Drosophila species other than Drosophila melanogaster. Nature, 310, 330-332.

Daniels S.B., Strausbaugh L.D., Ehrman L., Armstrong R., 1984. Sequences homologous to $\mathbf{P}$ elements occur in Drosophila paulistorum. Proc. Nat. Acad. Sc. (U.S.A.), 81, 6794-6797.

Daniels S.B., Strausbaugh L.D., 1985. The distribution of $\mathrm{P}$ element sequences in Drosophila : the willistoni and saltans species groups. J. of Mol. Evol. (sous presse).

Engels W.R., 1983. The P family of transposable elements in Drosophila. Ann. Rev. Genet., 17, 315-344.

HaCKman W., 1982. The relation between the genera Scaptomyza and Drosophila (Diptera, Drosophilidae). Ann. Ent. Fenn., 48, 97-104.

Kidwell M., 1983. Evolution of hybrid dysgenesis determinants in Drosophila melanogaster. Proc. Nat. Acad. Sci. (U.S.A.), 80, 1655-1659.

Kidwell M.G., Kidwell J.F., Sved J.A., 1977. Hybrid dysgenesis in Drosophila melanogaster : a syndrome of aberrant traits including mutation, sterility and male recombination. Genetics, 86, 813-833.

Martin G., WiernasZ D., Shedl P., 1983. Evolution of Drosophila repetitive-dispersed DNA. J. Mol. Evol., 19, 203-213.

O'HARE K., Rubin G.M., 1983. Structures of $\mathbf{P}$ transposable elements and their site of insertion and excision in the Drosophila melanogaster genome. Cell, 34, 25-35.

Tchen P., AnXolabehere D., Nouaud D., Périquet G., 1985. Hybridization on squashed flies : a method to detect gene sequences in single Drosophila individuals. Anal. Biochem. (sous presse).

THROCKMORTON L.H., 1985. The phylogeny, ecology and geography of Drosophila. In : KING R.C. (ed.), Handbook of Genetics, 421-469, Plenum Press, New York. 